\title{
ARTÍCULOS
}

\section{Contenido de carbono total en los componentes de especies arbóreas y arbustivas en áreas con diferente uso, en el matorral espinoso tamaulipeco, en México}

\author{
Total carbon content of the components of trees and shrubs species in areas with \\ different use, in tamaulipan thornscrub, Mexico \\ José Israel Yerena Yamallel a*, Javier Jiménez Pérez a, \\ Oscar Alberto Aguirre Calderón a , Eduardo Javier Treviño Garza a
}

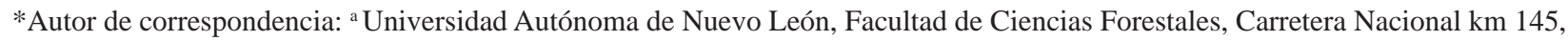
C.P. 67700, Linares, Nuevo León, México, tel.: 01821212 4895, yamallel@hotmail.com

\begin{abstract}
SUMMARY
Total carbon content coefficients were generated for basal biomass units for each of the components (stem, branches, small branches, leaves and bark) of trees and shrubs species in three areas with different historical use (traditional agriculture, extensive foraging and clearcut) on tamaulipan thornscrub, which will allow estimating carbon stored in this type of ecosystem. The total carbon content expressed as a percentage of biomass was determined with the Solids TOC Analyzer equipment that analyzes the total carbon contents in solid samples by complete combustion. By an ANOVA, we found a highly significant interaction between components and species; thus proving that the total carbon content of biomass components depends on the species. Highly significant differences were found among the components of all the species; the stem component (45.93 $\pm 0.18 \%)$, branches $(45.73 \pm 0.17 \%)$ and small branches (45.70 $\pm 0.19 \%$ ) showed similar behavior in the total carbon content; demonstrating differences when Tukey test was used, where the highest value $(48.14 \pm 0.30 \%)$ was presented by the carbon content of leaves and the lowest by bark $(42.08 \pm 0.21 \%)$.
\end{abstract}

Key words: carbon content, tamaulipan thornscrub.

\section{RESUMEN}

Se generaron coeficientes de contenido de carbono total por unidad de biomasa, para cada uno de los componentes (tallo, ramas, ramillas, hojas y corteza) de especies arbóreas y arbustivas en tres áreas con diferente uso histórico (agricultura tradicional, ganadería extensiva y matarasa) en el matorral espinoso tamaulipeco, los cuales permitirán estimar el carbono almacenado en este tipo de ecosistema. El contenido de carbono total expresado como porcentaje de la biomasa, se determinó con el equipo Solids TOC Analyzer que analiza los contenidos de carbono total en muestras sólidas mediante combustión completa. A través del ANDEVA, se encontró una interacción altamente significativa entre los componentes y especies, resultando que contenido de carbono total en los componentes de la biomasa depende de la especie. Se encontraron diferencias altamente significativas entre los componentes de todas las especies, los componentes: tallos $(45,93 \pm 0,18 \%)$, ramas $(45,73 \pm 0,17 \%)$ y ramillas $(45,70 \pm 0,19 \%)$ mostraron comportamiento similar en el contenido de carbono total, resultando diferencias mediante la prueba de Tukey con el contenido de carbono de las hojas, éste con el valor más alto $(48,14 \pm 0,30 \%)$ y la corteza con el menor $(42,08 \pm 0,21 \%)$.

Palabras clave: contenido de carbono, matorral espinoso tamaulipeco.

\section{INTRODUCCIÓN}

Existe una gran riqueza de especies forestales en los distintos tipos de vegetación, las cuales contribuyen a la producción de oxígeno y captura de carbono. En México existen diversos trabajos científicos sobre captura de carbono en diferentes tipos de vegetación o ecosistemas (de Jong et al. 1999, Ordóñez y Masera 2001, Masera et al. 2001, Ordóñez et al. 2008, Návar 2008), pero aún no se cuenta con información detallada sobre el contenido de carbono de las especies forestales. Esto complementaria en estimaciones más precisas de los almacenes de carbono en proyectos forestales para proyectos de reducción de las emisiones ocasionadas por deforestación y degradación de los bosques (REDD + ).

El dióxido de carbono $\left(\mathrm{CO}_{2}\right)$ atmosférico al incorporarse a los procesos metabólicos de las plantas mediante la fotosíntesis (Montoya et al. 1995), participa en la composición de todas las estructuras (follaje, ramas, raíces y tronco) necesarias para que la planta pueda desarrollarse (Ordóñez et al. 2008). Durante el tiempo en que el carbono se encuentra en alguna estructura del árbol y hasta que es 
liberado nuevamente al suelo o a la atmósfera se considera almacenado (Ordóñez y Masera 2001).

La variación del contenido de carbono en los tejidos del árbol ha sido escasamente documentada en México. En las estimaciones de la cantidad de carbono almacenado para biomasa aérea se asume generalmente el valor de la fracción de carbono en materia seca de un $50 \%$, sin diferenciar especies (IPCC 1996, Brown 1999, Houghton et al. 1999). No obstante, diferentes estudios denotan la variabilidad del contenido de carbono según la especie y tejido del árbol (Lin et al. 2002, Peri et al. 2004, Gayoso y Guerra 2005, Fonseca et al. 2011).

Uno de los estudios más extensos realizados en Australia por Gifford (2000), señala que el contenido de carbono en componentes leñosos depende de la proporción de compuestos como la lignina y minerales inorgánicos. Según esto, como los contenidos de lignina y minerales difieren entre los distintos tejidos del árbol, es esperable que los diversos componentes del árbol presenten distintos contenidos de carbono. Diversos autores (Ascencio 1982, Peñuelas y Lluisá 2003) mencionan que la variabilidad en el contenido de carbono en las hojas depende de las características anatómicas de estas, de los procesos fisiológicos como la fotorespiración, carboxilación y oxigenación, del balance o razón entre las actividades de las enzimas y de los cambios de la temperatura del ambiente.

Es necesario iniciar trabajos tendientes a establecer las bases metodológicas para la medición del carbono capturado y la inventarización de esta variable, con fines de certificación, como una alternativa de generación de recursos económicos para los pobladores del área. Por lo tanto, la hipótesis que se planteó es que los componentes de la biomasa aérea tallo, ramas, ramillas, hojas y corteza de las especies arbóreas y arbustivas en el matorral espinoso tamaulipeco presentan diferencias en el contenido de carbono total.

El objetivo de la presente investigación es generar coeficientes de contenido de carbono total por unidad de biomasa aérea base peso seco para cada uno de los componentes (tallo, ramas, ramillas, hojas y corteza) de las especies leñosas representativas en áreas con diferente uso en el matorral espinoso tamaulipeco.

\section{MÉTODOS}

Áreas de estudio. El estudio se realizó en la reserva ecológica del Matorral - Escuela de la Facultad de Ciencias Forestales de la Universidad Autónoma de Nuevo León y en dos áreas contiguas, con vegetación secundaria y condiciones similares (clima, suelo, altitud y pendiente). El clima es (A) C (x) (w») a (e) correspondiente a un clima extremoso semi-cálido - sub húmedo básicamente debido a la posición geográfica en la zona subtropical de altas presiones, con influencia de los vientos alisios húmedos y vientos fríos del norte durante el invierno. La precipitación pluvial anual oscila entre 400 y $1.850 \mathrm{~mm}$, con un valor promedio de $805 \mathrm{~mm}$ y desviación estándar de $260 \mathrm{~mm}$.
La temperatura promedio anual es de $22,4^{\circ} \mathrm{C}$ con temperaturas mayores a las $40^{\circ} \mathrm{C}$ en verano e inferiores a $0^{\circ} \mathrm{C}$ en invierno. Los suelos de la zona corresponden a un típico vertisol de origen aluvio-coluvial. Éstas se encuentran en una fracción del Matorral Espinoso Tamaulipeco de la Planicie Costera del Golfo en el municipio de Linares, Nuevo León, México, situado entre las coordenadas $25^{\circ} 09^{\prime}$ y $24^{\circ}$ $33^{\prime} \mathrm{N}$, y $99^{\circ} 54^{\prime}$ y $99^{\circ} 07^{\prime} \mathrm{O}$, en una altitud de $350 \mathrm{~m}$ s.n.m.

Las áreas de estudio fueron: 1) agricultura tradicional, área desmontada con maquinaria para la práctica agrícola, cultivo de maíz y sorgo de temporal, durante un periodo aproximado de cinco años. Este sitio tenía 22 años sin aprovechamiento. 2) Ganadería extensiva, superficie influenciada por la extracción selectiva de sus componentes para uso de leña, estantes y madera de construcción, además de la acción de ramoneo y pastoreo del ganado. Desde hace 27 años es reserva natural de la Facultad de Ciencias Forestales, la cual tiene como objetivo la enseñanza e investigación. 3) Matarasa, superficie desmontada hace 26 años con fines de investigación (Foroughbakhch y Heiseke 1990) utilizando la técnica del cadeneo (consiste en arrastrar una cadena de ancla de barco de 40 a 60 metros de largo que pesa 4 a $5 \mathrm{Mg}$, jalada por dos tractores de oruga). En este sitio no se realizó ninguna actividad silvoagropecuaria. La selección de estos sitios se debió a que el presente trabajo se realizó de forma paralela a estudios de recuperación de diversidad de especies y de reservas de carbono en el matorral con diferentes tipos de intervención.

Metodología de campo. De acuerdo a investigaciones realizadas en las áreas de estudio, se seleccionaron en cada sitio las cinco especies leñosas con mayor peso ecológico (Alanís et al. 2008, Jiménez et al. 2009) para la presente investigación (cuadro 1).

Diseño de muestreo y tamaño de la muestra. El método consideró la tabla de frecuencias de clases diamétricas para determinar el número de individuos a muestrear por especie, asegurando al menos un individuo de cada especie por cada clase diamétrica con frecuencia de al menos tres individuos. Es importante destacar que el presente trabajo se realizó de forma paralela a trabajos de evaluación de la biomasa aérea de dichas especies y que el muestreo de estos individuos se realizó de forma selectiva, considerando aspectos de no variación de exposición y libres de competencia por luz.

Colecta de las muestras. Una vez elegidos los individuos se realizaron dos tipos de mediciones. Unas con el individuo en pie y otras una vez volteado. Previo al volteo de los individuos se registró para cada árbol: número, especie, diámetro a $0,1 \mathrm{~m}$ y altura total.

Identificado el árbol o arbusto a procesar, se señaló la zona de caída de este y se determinó la zona de procesado con el fin de habilitarla para el trabajo. A continuación se describen los pasos realizados: a) volteo del individuo, realizando el corte lo más cerca posible del suelo; b) desrame 
Cuadro 1. Especies seleccionadas por sitio.

Selected species per site.

\begin{tabular}{lll}
\hline Nombre del Sitio & \multicolumn{1}{c}{ Nombre científico } & Nombre común \\
\hline Agricultura tradicional & Cercidium macrum I.M. Johnst. & Palo verde \\
Agricultura tradicional & Acacia rigidula Benth. & Gavia \\
Agricultura tradicional & Eysenhardtia texana Scheele & Vara dulce \\
Agricultura tradicional & Cordia boissieri A. DC. & Anacahuita \\
Agricultura tradicional & Acacia farnesiana Willd. & Huizache \\
Ganadería extensiva & Diospyros texana Scheele & Chapote \\
Ganadería extensiva & Acacia rigidula & Gavia \\
Ganadería extensiva & Eysenhardtia texana & Vara dulce \\
Ganadería extensiva & Havardia pallens Britton et Rose & Tenaza \\
Ganadería extensiva & Forestiera angustifolia Torr. & Panalero \\
Matarasa & Diospyros texana & Chapote \\
Matarasa & Acacia rigidula & Gavia \\
Matarasa & Bumelia celastrina H.B.K. & Coma \\
Matarasa & Havardia pallens & Tenaza \\
Matarasa & Acacia schaffneri F.J. Herm. & Huizache chino \\
\hline
\end{tabular}

y trozado, los árboles y arbustos se dividieron en sus componentes aéreos: fuste, ramas, ramillas ( $<1 \mathrm{~cm}$ diámetro) y hojas; c) colecta de muestras; fuste: se seleccionaron las muestras de madera de la sección radial a 0,10 m, se empaquetó en una bolsa de papel y se identificó la especie, número de individuo y sitio (esta nomenclatura se utilizó para todas las muestras); corteza: se colectó una porción de la sección donde se tomó la muestra de madera del fuste; ramas: se tomaron muestras a partir de $1 \mathrm{~cm}$ de diámetro en los cuatro puntos cardinales de la copa; ramillas: se tomaron muestras menores de $1 \mathrm{~cm}$ de diámetro en los cuatro puntos cardinales de la copa; hojas: se obtuvieron muestras en los cuatro puntos cardinales de la copa; las muestras que se tomaron de los cuatro puntos cardinales se mezclaron y se tomaron como una sola muestra por componente y por árbol.

Metodología de laboratorio. Colectadas las muestras en campo se procedió a pesar cada una de éstas en el laboratorio y posteriormente se colocaron en la estufa de secado a $105^{\circ} \mathrm{C}$ de temperatura, hasta obtener un peso constante. Cada muestra se trituró en un molino pulverizador, depositándolas en bolsas de plástico previamente identificadas.

El contenido de carbono total se determinó con un equipo analítico denominado Solids TOC Analyzer (este aparato puede medir el carbono orgánico total o carbono total, en este estudio se utilizó para carbono total) de $\mathrm{O} \cdot 1 \cdot$ Analytical; este determina los contenidos de carbono total en muestras sólidas mediante combustión completa, a una temperatura de $900^{\circ} \mathrm{C}$, los gases producto de la com- bustión son medidos a través de un detector infrarrojo no dispersivo que contabiliza las moléculas de carbono contenidas en estos gases.

Se realizaron tres análisis sobre una misma muestra de cada uno de los componentes aéreos de las especies, generando un promedio. En total se analizaron 675 submuestras de 225 muestras (cinco componentes por 45 individuos) de 45 individuos (tres individuos por especie presente en cada sitio).

Análisis estadístico. El análisis de los datos de laboratorio se realizó mediante el programa estadístico Statistica ${ }^{\circledR}$ versión 7.1. Para identificar diferencias significativas en el carbono total entre los componentes de la biomasa aérea de las especies y si éste es independiente o no de las especies, se realizó un análisis de varianza (ANDEVA) con un nivel de significancia del $5 \%(P \leq 0,05)$. Se determinaron dos factores: especie y componente; dentro del factor especie hubo 15 niveles (cinco especies por cada sitio, se consideraron las especies que se repitieron en los sitios: Acacia rigidula en tres sitios, Eysenhardtia texana, Diospyros texana y Havardia pallens en dos sitios, como diferentes niveles), y para el factor componente cinco niveles (fuste, ramas, ramillas, hojas y corteza). De esta manera se agruparon los datos bajo un diseño experimental factorial 15 x 5 .

Cuando hubo efectos significativos, se realizó un ANDEVA $(P \leq 0,05)$ completamente aleatorio para el factor componente. Luego, cuando hubo significancia en esta prueba, se procedió a realizar un análisis de comparación de medias a través de la prueba de Tukey $(P \leq 0,05)$. 
Por otra parte, se realizó un ANDEVA factorial para determinar si existían efectos significativos entre los componentes de las especies en los diferentes sitios, donde se determinaron dos factores: el sitio, con tres niveles (agricultura tradicional, ganadería y matarasa) y los componentes de la biomasa aérea de las especies, con cinco niveles (corteza, hojas, ramas, ramillas y fuste), de tal manera que hubo 15 interacciones $(3 \times 5)$.

Realizado el ANDEVA factorial, cuando hubo significancia entre los diferentes niveles de los factores (componentes de las de especies y sitios), se procedió a realizar un ANDEVA completamente aleatorio para componentes, estableciéndose como tratamientos los 15 componentes de los tres sitios (cinco para agricultura tradicional, cinco para ganadería y cinco para matarasa). Cuando resultó significancia en esta prueba, se procedió a realizar la prueba de comparación de medias de Tukey $(P \leq 0,05)$.

\section{RESULTADOS}

Carbono total (\%) por componente por especie por sitio. Entre los diferentes niveles de los factores componen- te y especie hubo una interacción altamente significativa $(P<0,001)$, es decir, ambos factores no son independientes, por lo que el contenido de carbono total en los componentes de la biomasa aérea dependió de la especie (cuadro 2). El rango de variación del contenido de carbono total entre los componentes de las especies fue de 10,52 puntos porcentuales. El componente corteza de las especies Cordia boissieri, Cercidium macrum, Acacia farnesiana, Eysenhardtia texana, Acacia rigidula del sitio agricultura tradicional, Acacia rigidula, Eysenhardtia texana del sitio ganadería extensiva y Diospyros texana, Bumelia celastrina, Acacia rigidula del sitio matarasa, resultó con valores inferiores (desde 39,62 hasta 42,67\%); en contraparte, el componente hojas de las especies, Acacia farnesiana, Acacia rigidula del sitio agricultura tradicional, Havardia pallens, Eysenhardtia texana, Acacia rigidula, Forestiera angustifolia del sitio ganadería extensiva y Bumelia celastrina, Havardia pallens, Diospyros texana, Acacia rigidula, Acacia schaffneri del sitio matarasa, presentó el contenido de carbono total más alto de todos los componentes de las especies, con valores desde 47,32 hasta 50,14\% (cuadro 2).

Cuadro 2. Carbono total (\%) por componente por especie por sitio.

Total carbon (\%) per component per species per site.

\begin{tabular}{|c|c|c|c|c|}
\hline Componente & Especie & Sitio & Media \pm EE & Agrupación Tukey ${ }^{1}$ \\
\hline Corteza & Cordia boissieri & Agricultura tradicional & $39,62 \pm 0,16$ & a \\
\hline Corteza & Cercidium macrum & Agricultura tradicional & $39,90 \pm 0,17$ & $\mathrm{a}$ \\
\hline Corteza & Acacia farnesiana & Agricultura tradicional & $41,33 \pm 0,64$ & $a b$ \\
\hline Corteza & Diospyros texana & Matarasa & $41,44 \pm 0,43$ & abc \\
\hline Corteza & Bumelia celastrina & Matarasa & $41,71 \pm 0,71$ & abcd \\
\hline Corteza & Acacia rigidula & Ganadería extensiva & $41,74 \pm 0,43$ & abcd \\
\hline Corteza & Eysenhardtia texana & Ganadería extensiva & $42,03 \pm 0,57$ & abcde \\
\hline Corteza & Acacia rigidula & Matarasa & $42,06 \pm 0,62$ & abcde \\
\hline Corteza & Eysenhardtia texana & Agricultura tradicional & $42,32 \pm 0,43$ & abcdef \\
\hline Corteza & Acacia rigidula & Agricultura tradicional & $42,67 \pm 0,68$ & abcdefg \\
\hline Corteza & Havardia pallens & Matarasa & $43,07 \pm 0,88$ & bcdefgh \\
\hline Hojas & Cordia boissieri & Agricultura tradicional & $43,09 \pm 0,47$ & bcdefghi \\
\hline Corteza & Acacia schaffneri & Matarasa & $43,12 \pm 0,81$ & bcdefghi \\
\hline Corteza & Diospyros texana & Ganadería extensiva & $43,17 \pm 0,23$ & bcdefghij \\
\hline Corteza & Havardia pallens & Ganadería extensiva & $43,47 \pm 0,29$ & bcdefghijk \\
\hline Ramas & Acacia farnesiana & Agricultura tradicional & $43,57 \pm 0,68$ & bcdefghijkl \\
\hline Corteza & Forestiera angustifolia & Ganadería extensiva & $43,60 \pm 0,38$ & bcdefghijkl \\
\hline Ramillas & Acacia farnesiana & Agricultura tradicional & $43,81 \pm 0,02$ & bcdefghijklm \\
\hline Ramillas & Acacia rigidula & Ganadería extensiva & $44,22 \pm 0,34$ & bcdefghijklmn \\
\hline Tallo & Acacia schaffneri & Matarasa & $44,44 \pm 0,13$ & bcdefghijklmno \\
\hline Tallo & Acacia rigidula & Agricultura tradicional & $44,54 \pm 0,27$ & cdefghijklmno \\
\hline Ramas & Acacia schaffneri & Matarasa & $44,75 \pm 0,23$ & defghijklmno \\
\hline
\end{tabular}


Continuación Cuadro 2

\begin{tabular}{|c|c|c|c|c|}
\hline Componente & Especie & Sitio & Media $\pm \mathrm{EE}$ & Agrupación Tukey ${ }^{1}$ \\
\hline Ramillas & Acacia rigidula & Matarasa & $44,81 \pm 0,37$ & defghijklmno \\
\hline Ramillas & Eysenhardtia texana & Agricultura tradicional & $44,89 \pm 0,39$ & efghijklmno \\
\hline Tallo & Acacia rigidula & Ganadería extensiva & $44,89 \pm 0,23$ & efghijklmno \\
\hline Tallo & Acacia rigidula & Matarasa & $44,91 \pm 0,41$ & efghijklmnop \\
\hline Ramas & Acacia rigidula & Ganadería extensiva & $44,95 \pm 0,18$ & efghijklmnop \\
\hline Ramillas & Acacia rigidula & Agricultura tradicional & $44,97 \pm 0,18$ & efghijklmnop \\
\hline Ramillas & Acacia schaffneri & Matarasa & $44,99 \pm 0,28$ & efghijklmnopq \\
\hline Ramas & Eysenhardtia texana & Agricultura tradicional & $45,09 \pm 0,47$ & efghijklmnopqr \\
\hline Ramas & Acacia rigidula & Agricultura tradicional & $45,23 \pm 0,17$ & fghijklmnopqr \\
\hline Tallo & Diospyros texana & Ganadería extensiva & $45,49 \pm 0,70$ & ghijklmnopqrs \\
\hline Ramillas & Cercidium macrum & Agricultura tradicional & $45,50 \pm 0,34$ & ghijklmnopqrs \\
\hline Tallo & Diospyros texana & Matarasa & $45,51 \pm 0,55$ & ghijklmnopqrs \\
\hline Ramas & Acacia rigidula & Matarasa & $45,60 \pm 0,26$ & ghijklmnopqrs \\
\hline Tallo & Acacia farnesiana & Agricultura tradicional & $45,83 \pm 0,62$ & hijklmnopqrs \\
\hline Ramas & Diospyros texana & Ganadería extensiva & $45,84 \pm 0,08$ & hijklmnopqrs \\
\hline Ramas & Cercidium macrum & Agricultura tradicional & $45,85 \pm 0,86$ & hijklmnopqrs \\
\hline Tallo & Cercidium macrum & Agricultura tradicional & $45,87 \pm 0,51$ & hijklmnopqrs \\
\hline Ramillas & Diospyros texana & Matarasa & $45,87 \pm 0,53$ & hijklmnopqrs \\
\hline Ramillas & Cordia boissieri & Agricultura tradicional & $45,90 \pm 0,20$ & hijklmnopqrs \\
\hline Ramas & Diospyros texana & Matarasa & $45,94 \pm 0,79$ & hijklmnopqrs \\
\hline Ramillas & Diospyros texana & Ganadería extensiva & $46,20 \pm 0,30$ & hijklmnopqrst \\
\hline Ramillas & Havardia pallens & Ganadería extensiva & $46,21 \pm 0,32$ & hijklmnopqrst \\
\hline Ramas & Cordia boissieri & Agricultura tradicional & $46,22 \pm 0,49$ & ijklmnopqrst \\
\hline Tallo & Bumelia celastrina & Matarasa & $46,29 \pm 0,42$ & jklmnopqrst \\
\hline Ramillas & Havardia pallens & Matarasa & $46,41 \pm 0,59$ & klmnopqrst \\
\hline Tallo & Cordia boissieri & Agricultura tradicional & $46,43 \pm 0,15$ & klmnopqrst \\
\hline Ramas & Forestiera angustifolia & Ganadería extensiva & $46,46 \pm 0,36$ & klmnopqrstu \\
\hline Ramas & Eysenhardtia texana & Ganadería extensiva & $46,49 \pm 0,47$ & klmnopqrstu \\
\hline Ramillas & Eysenhardtia texana & Ganadería extensiva & $46,51 \pm 0,86$ & klmnopqrstu \\
\hline Ramas & Havardia pallens & Matarasa & $46,51 \pm 1,07$ & klmnopqrstu \\
\hline Hojas & Eysenhardtia texana & Agricultura tradicional & $46,54 \pm 0,40$ & klmnopqrstu \\
\hline Tallo & Eysenhardtia texana & Agricultura tradicional & $46,54 \pm 0,10$ & klmnopqrstu \\
\hline Hojas & Cercidium macrum & Agricultura tradicional & $46,55 \pm 1,25$ & klmnopqrstuv \\
\hline Ramas & Bumelia celastrina & Matarasa & $46,64 \pm 0,22$ & Imnopqrstuv \\
\hline Tallo & Eysenhardtia texana & Ganadería extensiva & $46,68 \pm 1,09$ & lmnopqrstuvw \\
\hline Tallo & Havardia pallens & Matarasa & $46,76 \pm 0,89$ & mnopqrstuvw \\
\hline Ramas & Havardia pallens & Ganadería extensiva & $46,81 \pm 0,47$ & mnopqrstuvw \\
\hline Hojas & Diospyros texana & Ganadería extensiva & $46,84 \pm 0,49$ & mnopqrstuvw \\
\hline Ramillas & Bumelia celastrina & Matarasa & $47,01 \pm 0,75$ & nopqrstuvwx \\
\hline Hojas & Diospyros texana & Matarasa & $47,32 \pm 0,60$ & nopqrstuvwx \\
\hline Tallo & Forestiera angustifolia & Ganadería extensiva & $47,39 \pm 0,75$ & opqrstuvwx \\
\hline Tallo & Havardia pallens & Ganadería extensiva & $47,45 \pm 0,27$ & opqrstuvwx \\
\hline Hojas & Acacia farnesiana & Agricultura tradicional & $48,06 \pm 0,37$ & pqrstuvwx \\
\hline
\end{tabular}




\begin{tabular}{|c|c|c|c|c|}
\hline Componente & Especie & Sitio & Media \pm EE & Agrupación Tukey ${ }^{1}$ \\
\hline Ramillas & Forestiera angustifolia & Ganadería extensiva & $48,13 \pm 0,65$ & qrstuvwx \\
\hline Hojas & Havardia pallens & Ganadería extensiva & $48,23 \pm 0,87$ & rstuvwx \\
\hline Hojas & Eysenhardtia texana & Ganadería extensiva & $48,58 \pm 0,36$ & stuvwx \\
\hline Hojas & Acacia rigidula & Ganadería extensiva & $49,10 \pm 0,41$ & tuvwx \\
\hline Hojas & Acacia rigidula & Matarasa & $49,27 \pm 0,56$ & tuvwx \\
\hline Hojas & Acacia rigidula & Agricultura tradicional & $49,34 \pm 0,55$ & tuvwx \\
\hline Hojas & Bumelia celastrina & Matarasa & $49,59 \pm 0,07$ & uvwx \\
\hline Hojas & Havardia pallens & Matarasa & $49,69 \pm 0,22$ & vwx \\
\hline Hojas & Forestiera angustifolia & Ganadería extensiva & $49,82 \pm 0,62$ & wx \\
\hline Hojas & Acacia schaffneri & Matarasa & $50,14 \pm 0,28$ & $\mathrm{x}$ \\
\hline
\end{tabular}

${ }^{1}$ Letras iguales son estadísticamente similares $(P \leq 0,05)$.

$\mathrm{EE}=$ error estándar de la muestra.

Carbono total (\%) por componente de todas las especies. Se obtuvieron diferencias altamente significativas entre los porcentajes promedio de los componentes del conjunto de todas las especies $(P<0,001)$ (cuadro 3$)$. El componente corteza presentó el valor inferior de carbono (42,08 \%) respecto de los otros componentes $(P \leq 0,05)$. Los componentes tallo, ramas y ramillas no presentaron diferencias significativas entre sí $(P>0,05)$, formando un grupo intermedio. El componente hojas presentó el contenido de carbono total más alto $(48,14 \%)$ de todos los componentes $(P \leq 0,05)$.

Carbono total (\%) por componente por sitio. Se estimó que existen diferencias altamente significativas $(P<0,001)$ entre los componentes de la biomasa aérea de los sitios (cuadro 4). El componente corteza en los tres sitios resultó con valores inferiores al resto de los componentes $(P \leq 0,05)$, variando de 41,17 a $42,80 \%$, mientras que los componentes tallo, ramas y ramillas en los tres sitios no presentaron diferencia significativa entre sí $(P \leq 0,05)$, con valores de 45,01 a 46,38 \%. Las hojas en los sitios matarasa y ganadería extensiva, presentaron valores superiores a los demás componentes, el rango fue de 48,51 a 49,20\% $(P \leq 0,05)$.

Cuadro 3. Carbono total por componente de todas las especies (\%). Total carbon per component of all species (\%).

\begin{tabular}{|c|c|c|}
\hline Componente & Media $\pm \mathrm{EE}$ & Agrupación Tukey ${ }^{1}$ \\
\hline Corteza & $42,08 \pm 0,21$ & $\mathrm{a}$ \\
\hline Ramillas & $45,70 \pm 0,19$ & $\mathrm{~b}$ \\
\hline Ramas & $45,73 \pm 0,17$ & $\mathrm{~b}$ \\
\hline Tallo & $45,93 \pm 0,18$ & $\mathrm{~b}$ \\
\hline Hojas & $48,14 \pm 0,30$ & c \\
\hline
\end{tabular}

${ }^{1}$ Letras iguales son estadísticamente similares $(P \leq 0,05)$.

$\mathrm{EE}=$ error estándar de la muestra.

\section{DISCUSIÓN Y CONCLUSIONES}

Se observa que hay un amplio rango de variación en los coeficientes de contenido de carbono total de los componentes de la biomasa aérea de las especies, desde 39,62 \% en la corteza de Cordia boissieri hasta 50,14 \% en las hojas de Acacia schaffneri, resulta, en la mayoría de los casos (a excepción de este último componente) inferior al valor por defecto de $50 \%$ sugerido por el Grupo Intergubernamental de Expertos sobre el Cambio Climático (IPCC 1996).

Los componentes tallos, ramas y ramillas de todas las especies muestran un comportamiento similar en el contenido de carbono total, resultando menor que el de las hojas. Este último componente presenta el valor más alto y la corteza el menor de todos. Por lo tanto, la hipótesis planteada se confirma. Lo anterior se atribuye a que en las hojas se inicia el proceso de fotosíntesis (absorción de $\mathrm{CO}_{2}$ ), siendo estos órganos sitios con elevada concentración de carbohidratos. En las ramas se realiza la diferenciación y elongación de los meristemos que darán origen a los órganos reproductivos o vegetativos, lo que origina que el carbono se almacene en forma de carbohidratos o compuestos de reserva (Watson y Casper 1984). En contraste, en el tallo la mayoría del carbono es celulosa y lignina. El contenido de carbono promedio de los componentes tallo, ramas, ramillas y corteza de las especies de este estudio es más bajo que los determinados por Francis (2000), Petre et al. (2006), Díaz (2008) y Fonseca et al. (2012). Dicha diferencia se atribuye a que los componentes de las especies de dichos estudios contienen mayores contenidos de elementos químicos (celulosa, hemicelulosa y lignina) que los de este estudio, compuestos que tienen carbono.

En el sitio de ganadería extensiva, considerado como un sistema permanente y con mayor crecimiento, los componentes de la biomasa presentan los valores mayores de carbono, a excepción de la corteza. En el sitio de agricultu- 
Cuadro 4. Carbono total por componente por sitio (\%).

Total carbon per component per site (\%).

\begin{tabular}{lllc}
\hline Componente & \multicolumn{1}{c}{ Sitio } & Media \pm EE & Agrupación Tukey ${ }^{1}$ \\
\hline Corteza & Agricultura tradicional & $41,17 \pm 0,37$ & a \\
Corteza & Matarasa & $42,28 \pm 0,33$ & ab \\
Corteza & Ganadería extensiva & $42,80 \pm 0,25$ & $\mathrm{~b}$ \\
Ramillas & Agricultura tradicional & $45,01 \pm 0,21$ & $\mathrm{c}$ \\
Ramas & Agricultura tradicional & $45,19 \pm 0,33$ & $\mathrm{~cd}$ \\
Tallo & Matarasa & $45,58 \pm 0,31$ & $\mathrm{~cd}$ \\
Ramillas & Matarasa & $45,82 \pm 0,30$ & $\mathrm{~cd}$ \\
Tallo & Agricultura tradicional & $45,84 \pm 0,24$ & $\mathrm{~cd}$ \\
Ramas & Matarasa & $45,89 \pm 0,30$ & $\mathrm{~cd}$ \\
Ramas & Ganadería extensiva & $46,11 \pm 0,22$ & $\mathrm{~cd}$ \\
Ramillas & Ganadería extensiva & $46,25 \pm 0,39$ & $\mathrm{~cd}$ \\
Tallo & Ganadería extensiva & $46,38 \pm 0,38$ & $\mathrm{~cd}$ \\
Hojas & Agricultura tradicional & $46,72 \pm 0,62$ & $\mathrm{~d}$ \\
Hojas & Ganadería extensiva & $48,51 \pm 0,34$ & $\mathrm{e}$ \\
Hojas & Matarasa & $49,20 \pm 0,31$ & $\mathrm{e}$ \\
\hline
\end{tabular}

${ }^{1}$ Letras iguales son estadísticamente similares $(P \leq 0,05)$.

$\mathrm{EE}=$ error estándar de la muestra.

ra tradicional sometido a la mayor intervención y en proceso de recuperación, los componentes ramas, ramillas, hojas y corteza resultan con los valores menores de carbono.

En un análisis de las complicaciones de tener estimaciones de contenido de carbono por componente, se tomaron los datos de biomasa $\left(\mathrm{Mg} \mathrm{ha}^{-1}\right)$ que reportan Navar et al. (2002) para el matorral espinoso tamaulipeco, calculando la cantidad de carbono estimada con los valores de porcentaje de carbono del IPCC (1996) y Brown (1999) y, a su vez, con los de este estudio. De ello resulta una diferencia de 1,5 $\mathrm{Mg} \mathrm{ha}^{-1}$ (cuadro 5), con lo que se podría reflejar una sobrestimación significativa al emplear un valor estándar de $50 \%$ de carbono.

El matorral espinoso tamaulipeco es sumidero de dióxido carbono atmosférico debido a su capacidad de almacenar y conservar este. Los resultados de este trabajo sir- ven para las estimaciones de emisiones de $\mathrm{CO}_{2}$ a causa de la deforestación y degradación, como datos más precisos y confiables, lo cual permite un mayor acercamiento a conocer cómo son los comportamientos del flujo de carbono en este ecosistema forestal.

\section{AGRADECIMIENTOS}

A la Facultad de Ciencias Forestales de la Universidad Autónoma de Nuevo León, por haberme aceptado en su programa de Maestría en Ciencias Forestales y al Consejo Nacional de Ciencia y Tecnología (CONACYT) por la beca otorgada al primer autor para realizar dicha Maestría. Asimismo, se agradece al editor de la revista y a los árbitros anónimos por las sugerencias y recomendaciones que enriquecieron el manuscrito.

Cuadro 5. Cantidad de carbono en el matorral espinoso tamaulipeco. Amount of carbon in tamaulipan thornscrub.

\begin{tabular}{|c|c|c|c|c|c|}
\hline \multirow{3}{*}{ Componente } & \multirow{3}{*}{$\begin{array}{c}\text { Navar et al. (2002) } \\
\text { Biomasa } \\
\text { Mg ha }^{-1}\end{array}$} & \multirow{2}{*}{\multicolumn{2}{|c|}{$\frac{\text { IPCC (1996) y Brown (1999) }}{\text { Carbono }}$}} & \multicolumn{2}{|c|}{ En este estudio } \\
\hline & & & & \multicolumn{2}{|c|}{ Carbono } \\
\hline & & $\%$ & $\mathrm{Mg} \mathrm{ha}^{-1}$ & $\%$ & $\mathrm{Mg} \mathrm{ha}^{-1}$ \\
\hline Ramas & 24,44 & 50 & 12,22 & 45,73 & 11,17 \\
\hline Tallo & 9,80 & 50 & 4,90 & 45,93 & 4,50 \\
\hline Hojas & 2,51 & 50 & 1,25 & 48,14 & 1,20 \\
\hline Total & 36,75 & - & 18,37 & - & 16,87 \\
\hline
\end{tabular}




\section{REFERENCIAS}

Alanís E, J Jiménez, O Aguirre, E Treviño, E Jurado, M González. 2008. Efecto del uso del suelo en la fitodiversidad del matorral espinoso tamaulipeco. Ciencia UANL 11(1): 56-62.

Ascencio J. 1982. Mecanismos fotosintéticos en plantas con fotosíntesis intermedia C3-C4 y en plantas acuáticas. Revista de la Facultad de Agronomía (Maracay) 12(3-4): 267-282.

Brown S. 1999. Guidelines for inventorying and monitoring carbon offsets in forest-based projects. Winrock International for the World Bank. Arlington, Virginia, E.U.A. 11 p. Consultado 10 dic. 2010. Disponible en http://www.winrock. org/ecosystems/

De Jong BH, MA Cairns, PK Haggerty, MN Ramírez, GS Ochoa, VJ Mendoza, EM González, MI March. 1999. Land-use change and carbon flux between 1970s and 1990s in the central highlands of Chiapas, México. Environmental Management 23(3): 373-385.

Díaz J. 2008. Contenido de carbono total en los componentes de la biomasa aérea de las especies representativas del bosque de pino - encino en la sierra madre oriental. Tesis de Maestría. Linares, Nuevo León, México. Facultad de Ciencias Forestales, Universidad Autónoma de Nuevo León. 60 p.

Fonseca W, JM Rey-Benayas, FE Alice. 2011. Carbon accumulation in the biomass and soil of different aged secondary forests in the humid tropics of Costa Rica. Forest Ecology and Management 262(8): 1400-1408. DOI: 10.1016/j.foreco.2011.06.036.

Fonseca W, FE Alice, JM Rey-Benayas. 2012. Carbon accumulation in aboveground and belowground biomass and soil of different age native forest plantations in the humid tropical lowlands of Costa Rica. New Forest 43: 197-211. DOI: 10.1007/s11056-011-9273-9.

Foroughbakhch R, D Heiseke. 1990. Manejo silvícola del matorral: raleo, enriquecimiento y regeneración controlada. Reporte Científico No. 19. Linares, Nuevo León, México. Facultad de Ciencias Forestales, Universidad Autónoma de Nuevo León. 6 p.

Francis J. 2000. Estimating Biomass and Carbon Content of Saplings in Puerto Rican Secondary Forests. Caribbean Journal of Science 36(3-4): 346-350.

Gayoso J, J Guerra. 2005. Contenido de carbono en la biomasa aérea de bosques nativos en Chile. Bosque 26(2): 33-38.

Gifford R. 2000. Carbon contents of above-ground tissues of forest and woodland trees. Australian Greenhouse Office, National Carbon Accounting System, Technical Report N ${ }^{\circ}$ 22, Canberra. 27 p. Consultado 10 dic. 2010. Disponible en http://pandora.nla.gov.au/tep/23322/

Houghton RA, JL Hackler, KT Lawrence. 1999. The U.S. carbon budget: Contributions from land-use change. Science 285: 574-577.

IPCC (Intergovernmental Panel on Climate Change). 1996. Chapter 5: Land Use Change \& Forestry. Greenhouse Gas
Inventory Reference Manual. IPCC Guidelines for National Greenhouse Gas Inventories, Revised Version. London. 3: 57 p. Consultado 10 dic. 2010. Disponible en http://www. ipcc.ch/

Jiménez J, E Alanís, O Aguirre, M Pando, M González. 2009. Análisis sobre el efecto del uso del suelo en la diversidad estructural del matorral espinoso tamaulipeco. Madera y Bosques 15(3): 5-20

Lin YJ, CP Liu, JC Lin. 2002. Measurement of specific gravity and carbon content of important timber species in Taiwan. Journal of Forest Science 17(3): 291-299.

Masera O, A Cerón, A Ordóñez. 2001. Forestry mitigation options for Mexico: finding synergies between national sustainable development priorities and global concerns. Mitigation Adaptation Strategies Global Change 6: 291-312.

Montoya G, L Soto, B de Jong, K Nelson, P Farias, T Pajal, J Taylor, R Tipper. 1995. Desarrollo Forestal Sustentable: captura de carbono en las zonas Tzeltal y Tojolabal del estado de Chiapas. Distrito Federal, México. Instituto Nacional de Ecología. Cuadernos de Trabajo 4. 50 p.

Návar J, E Mendez, V Dale. 2002. Estimating stand biomass in the Tamaulipan thornscrub of northeastern Mexico. Annals of Forest Sciences 59: 813-821. DOI: 10.1051/forest:2002079.

Návar J. 2008. Carbon fluxes resulting from land-use changes in the Tamaulipan thornscrub of northeastern Mexico. Carbon Balance and Management 3:6. DOI: 10.1186/1750-06803-6.

Ordóñez A, O Masera. 2001. La captura de carbono ante el cambio climático. Madera y Bosques 7(1): 3-12.

Ordóñez A, B de Jong, F García, F Aviña, J Pérez, G Guerrero, R Martínez, O Masera. 2008. Carbon content in vegetation, litter, and soil under 10 different land-use and land-cover classes in the Central Highlands of Michoacan, México. Forest Ecology and Management 255(7): 2074-2084.

Peñuelas J, J Lluisá. 2003. Emisiones biogénicas de COVs y cambio global ¿Se defienden las plantas contra el cambio climático? Ecosistemas 1: 1-7.

Peri P, M Viola, G Martínez. 2004. Estimación de biomasa y secuestro de carbono en bosques nativos de ñire (Nothofagus antarctica) en Patagonia Sur. Publicación Técnica Forestal No 24, Convenio INTA-UNPA-CAP. Argentina. 9 p. Consultado 3 dic. 2010. Disponible en http://www.inta.gov.ar/ santacruz/

Petre A, U Ola, S Ali, A Reynero. 2006. Alternativas de sustentabilidad del bosque nativo del Espinal, Área Captura de Carbono. Proyectos de Investigación Aplicada a los Recursos Forestales Nativos N4085-AR. Secretaria de Ambiente y Desarrollo Sustentable de la Nación. Argentina. 15 p. Consultado 3 dic. 2010. Disponible en http://www.ambiente.gov.ar/

Watson MA, BB Casper. 1984. Morphogenetic constraints on patterns of carbon distribution in plants. Annual Review of Ecology and Systematics 15: 233-258. 